\title{
A Case Report on Functional Rehabilitation of Post-Operative Tendoachilles
}

\author{
Prasad B. Risaldar ${ }^{1}$, Laukik Vaidya ${ }^{1}$ and Kiran Kumar ${ }^{2 *}$ \\ ${ }^{1}$ Department of Musculoskeletal Physiotherapy, Ravi Nair Physiotherapy College, Datta \\ Meghe Institute of Medical Sciences, Sawangi (Meghe), Wardha, Maharashtra, India. \\ ${ }^{2}$ Associate Professor, Head of the department of musculoskeletal physiotherapy, Ravi Nair Physiotherapy College, \\ DattaMeghe Institute of Medical Sciences (Deemed to be university) Sawangi (Meghe), Wardha, Maharashtra
}

\begin{abstract}
The Achilles tendon acute rupture (ATR) is a prolonged injury, since the operative reconstruction marks the beginning of a lengthy period of time. Duration of recuperation, a significant feature of post-surgical recovery is in the treatment of such injuries, the aim of early restoration of the level of pre-injury operation, without raising the risk of rupture or elongation of tendons. Despite the growing number of available RCTs and feedback, there is still no general agreement on the most desirable protocol. In addition, the available evidence is routinely ignored. A 40-year-old farmer reported with history of accidental injury by a plough to left foot. There was sudden pain, bleeding following the injury and was not able to stand on his own. The patient was unable to bear weight on the left foot and therefore was unable to take further action. Left tendoachilles tear Surgical repair of tendon followed by physical therapy intervention for functional rehabilitation. Physical therapy for 6 weeks led to early recovery of functional activities.
\end{abstract}

KEY WORDS: TENDON REPAIR, SURGICAL REPAIR, FUNCTIONAL, REHABILITATION.

\section{INTRODUCTION}

The Achilles tendon acute rupture (ATR) is a prolonged injury, since the operative reconstruction marks the beginning of a lengthy period of time. Duration of recuperation, asignificant feature of post-surgical recovery is in the treatment of such injuries, the aim of early restoration of the level of pre-injury operation, without raising the risk of rupture or elongation of tendons (Brumann et al., 2014). Despite the growing number of available RCTs and feedback, there is still no general agreement on the most desirable protocol. In addition, the available evidence is routinely ignored (Brumann et al., 2014). To predict a decreased risk of re-rupture, surgical repair has been suggested to

Biosc Biotech Res Comm P-ISSN: 0974-6455 E-ISSN: 2321-4007

\section{crossey}

Identifiers and Pagination

Year: 2021 Vol: 14 No (6) Special Issue

Pages: $84-86$

This is an open access article under Creative

Commons License Attribn 4.0 Intl (CC-BY).

DOI: http://dx.doi.org/10.21786/bbrc/14.6.20 increase strength(Okoroha et al., 2020). Both treatment options-non-surgical closed methods, open-label surgical procedures or percutaneous repair methods-include cast use (Mandelbaum et al., 1995).

A matter of discussion remains the correct postoperative recovery technique for acute Achilles tendon rupture (Zhao et al., 2017). It's indeed crucial that patients recover during the first year after injury, as 1-year results predict whether long-term disabilities will remain or not (Zellers et al., 2019). Traditionally, a prolonged period of operational management has been accompanied by non-weight bearing (NWB) to prevent the dreaded complication of tendon re-rupture with rigid cast ankle immobilization (Lightsey et al., 2019). A number of articles from GBD studies reflect on this issue (James et al., 2020; James et al., 2017;Murray et al., 2020; Murray et al., 2020; Vos et al., 2020). Studies on tendon repair surgeries have been reported (Jawade et al., 1995).

Patient Information and observation: A 40-year-old farmer who was feeding his cattle's nearby his residence, accidently tripped on a plough lying near by leading to an

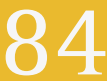


injury near the left foot. There was sudden pain, bleeding following the injury and was not able to stand on his own. He was taken to the rural hospital in 'Sawangi(Meghe), Wardha, Maharashtra(IN)' by hisneighbours, then there he was confirmed forachilles tendon cut,he was advised for immediate surgical left tendoachilles repair to prevent complications like re-rupture of the tendon. A reconstructive surgery was performed. A below knee slab was applied to prevent stretching of the tendon and to protect it. He was then referred to physiotherapy for further management of the repair and to help him for his activities of daily living.

Clinical findings: After the physical evaluation of the injured leg, it was discovered that owing to discomfort, the patient was unable to bear weight on the left foot and therefore was unable to take further action. Therefore, he was advised for surgical repair due to left tendoachilles rupture. Soon after the surgery the foot was kept in complete immobilization by the means of plaster cast with fingers left open for the movement for 6 weeks.

\begin{tabular}{|c|c|c|c|c|c|}
\hline Joint & Lt Active & Lt Passive & Rt Active & Rt Passive & Limitation \\
\hline \multicolumn{6}{|l|}{ Hip } \\
\hline Flexion & $0^{0}-60^{0}$ & $0^{0}-65^{0}$ & $0^{0}-65^{0}$ & $0^{0}-70^{0}$ & \multirow{12}{*}{$\begin{array}{l}\text { Cannot perform on left side } \\
\text { Cannot perform on left side } \\
\text { Cannot perform on left side } \\
\text { Cannot perform on left side }\end{array}$} \\
\hline Extension & $60^{0}-0^{0}$ & $65^{0}-0^{0}$ & $65^{0}-0^{0}$ & $70^{0}-0^{0}$ & \\
\hline Abduction & $0^{0}-35^{0}$ & $0^{0}-40^{0}$ & $0^{0}-30^{\circ}$ & $0-35^{0}$ & \\
\hline Adduction & $35^{0}-0^{0}$ & $40^{\circ}-0^{0}$ & $30^{\circ}-0^{0}$ & $35^{0}-0^{0}$ & \\
\hline \multicolumn{5}{|l|}{ Knee } & \\
\hline Flexion & $0^{0}-105^{0}$ & $0^{0}-110^{0}$ & $0^{0}-120^{0}$ & $0^{0}-125^{0}$ & \\
\hline Extension & $105^{0}-0^{0}$ & $110^{0}-0^{0}$ & $120^{0}-0^{0}$ & $125^{0}-0^{0}$ & \\
\hline \multicolumn{5}{|l|}{ Ankle } & \\
\hline Plantar Flexion & NA & NA & $00-450$ & $0-450$ & \\
\hline Doris Flexion & NA & NA & $0-150$ & $0-150$ & \\
\hline Inversion & NA & NA & $0-300$ & $0-300$ & \\
\hline Eversion & NA & NA & $0-200$ & $0-200$ & \\
\hline
\end{tabular}

\section{Physiotherapy Intervention}

\section{Short term goals}

1. To prevent respiratory complications

2. To reduce pain

3. To prevent oedema

4. To increase bed mobility

5. To prevent stiffness

\section{Long term goals}

1. To stand on his own

2. To perform his daily activities

3. To make him independent

4. To prevent re-injury

Phase 1: (0-6 weeks) (NWB)

Initially, the patient was taught finger movement to the affected lower limb. also the patient was advised spirometry to improve the respiratory function due to prolonged bed rest.Limb elevationwas given in order to reduce swelling. Passiverange of motion (PROM) for the unaffected limbs was given. To minimize the risk of developing pressure sores positioning was taught. Pelvic bridging was taught to the patient to reduce the pressure on the back and help the patient with his toileting activities and core strengthening will include static abdominals, unilateral bridging. Strengthening of upper limb, Non weight bearing with walker will be initiated with assistance.
Phase 2: (7- 9 weeks) (PWB)

Following the phase 1 exercise programme, the exercises were continued with added resistance with progressions. After the removal of cast, strengthening to the affected lower limb will be initiated, weight bearing will be initiated to the affected leg for up to $10-50 \%$ weight bearing.

Phase 3: (9-12 weeks) (TWB)

50-100\% weight bearing will be taught in phase 3 where the progression will be taught with the help of axillary crutches followed by full weight bearing. After the full weight bearing is achieved stair climbing and other complex activities are taught and challenged followed return to his daily activities.

\section{DISCUSSION}

In this case report we had a farmer with tendoachilles cut for whom a soft tissue reconstruction surgery was done, the rehabilitation objectives was formulated with regards to the surgery done and his nature of work, starting with walker to complete weight bearing with and without walking aid. All the exercises were done in three sessions of 10 repetitions of each exercise in every session, with the help of rehabilitation program he was able to resume his ADLs independently. The acute Achilles tendon rupture was paired with ankle immobilization for 6 weeks mostly as surgical procedure 
is performed. The typical recovery treatment involves rigid cast immobilization, normally for six weeks in a below-knee non-weight bearing rigid cast, accompanied by ankle joint mobilization and strength training. (4) A number of related studies were reviewed (Telang et al., 2020; Nikose et al., 2020; Phansopkar et al.,2020;). In this case, we delayed the weight bearing to 6 weeks to promote healing.

Limitation: Weight bearing was delayed to promote healing of the wound and to promote rupture of the wound.

\section{CONCLUSION}

Patient showed a great co-operation during the intervention period and now the subject is able to perform his daily activities. The outcome measures of physical therapy intervention progressed him with return to his normal functional tasks.

\section{REFERENCES}

Brumann, M., Baumbach, S.F., Mutschler, W. and Polzer, H., 2014. Accelerated rehabilitation following Achilles tendon repair after acute rupture-development of an evidence-based treatment protocol. Injury, 45(11), pp.1782-1790.

James, S.L., Castle, C.D., Dingels, Z.V., Fox, J.T., Hamilton, E.B., Liu, Z., Roberts, N.L., Sylte, D.O., Bertolacci, G.J., Cunningham, M. and Henry, N.J., 2020. Estimating global injuries morbidity and mortality: methods and data used in the Global Burden of Disease 2017 study. Injury Prevention, 26(Supp 1), pp.i125-i153.

Jawade, S., 2020. Post-operative physiotherapy rehabilitation in rare combined full thickness tear of supraspinatus and subscapularis tendon managed by arthroscopy with mini open Supraspinatus tendon repair: A Case report. European Journal of Molecular Et Clinical Medicine, 7(2), pp.1212-1219.

Latchoumi, T.P., Ezhilarasi, T.P. and Balamurugan, K., 2019. Bio-inspired weighed quantum particle swarm optimization and smooth support vector machine ensembles for identification of abnormalities in medical data. SN Applied Sciences, 1(10), pp.1-10.

Lightsey, H.M., Noback, P.C., Caldwell, J.M.E., Trofa, D.P., Greisberg, J.K. and Vosseller, J.T., 2019. Online physical therapy protocol quality, variability, and availability in Achilles tendon repair. Foot \& ankle specialist, 12(1), pp.16-24.

Mandelbaum, B.R., Myerson, M.S. and Forster, R., 1995. Achilles tendon ruptures: a new method of repair, early range of motion, and functional rehabilitation. The American Journal of Sports Medicine, 23(4), pp.392395.

Murray, C.J., Abbafati, C., Abbas, K.M., Abbasi, M., Abbasi-Kangevari, M., Abd-Allah, F., Abdollahi, M.,
Abedi, P., Abedi, A., Abolhassani, H. and Aboyans, V., 2020. Five insights from the global burden of disease study 2019. The Lancet, 396(10258), pp.1135-1159.

Murray, C.J., Aravkin, A.Y., Zheng, P., Abbafati, C., Abbas, K.M., Abbasi-Kangevari, M., Abd-Allah, F., Abdelalim, A., Abdollahi, M., Abdollahpour, I. and Abegaz, K.H., 2020. Global burden of 87 risk factors in 204 countries and territories, 1990-2019: a systematic analysis for the Global Burden of Disease Study 2019. The Lancet, 396(10258), pp.1223-1249.

Nikose, S.S., Nikose, D., Jain, S., Kekatpure, A., Saoji, K., Chaudhary, R. and Pisulkar, G., 2021. Determinants of regeneration and strength of hamstrings after anterior cruciate ligament reconstruction-fate of hamstring tendon. International Orthopaedics, pp.1-10.

Okoroha, K.R., Ussef, N., Jildeh, T.R., Khalil, L.S., Hasan, L., Bench, C., Zeni, F., Eller, E. and Moutzouros, V., 2020. Comparison of tendon lengthening with traditional versus accelerated rehabilitation after Achilles tendon repair: a prospective randomized controlled trial. The American journal of sports medicine, 48(7), pp.17201726.

Phansopkar, P., Athawale, V., Birelliwar, A., Naqvi, W. and Kamble, S., 2020. Post-operative rehabilitation in a traumatic rare radial nerve palsy managed with tendon transfers: a case report. The Pan African Medical Journal, 36.

Spencer, L., Chris, D., Zachary, V., Jack, T., Erin, B., Zichen, L., Nicholas, L., Roberts, S., Dillon, O., Nathaniel, J. and Kate, E., 2019. Global injury morbidity and mortality from 1990 to 2017: results from the Global Burden of Disease Study 2017.

Telang, P.A., Naqvi, W., Dhankar, S. and Jungade, S., 2020. Effect Of Manual Therapy (MET) Vs Conventional Therapy For Improving Tendo-Achilles (TA) Flexibility And Foot Posture In Children With Autism Spectrum Disorder.

Vos, T., Lim, S.S., Abbafati, C., Abbas, K.M., Abbasi, M., Abbasifard, M., Abbasi-Kangevari, M., Abbastabar, H., Abd-Allah, F., Abdelalim, A. and Abdollahi, M., 2020. Global burden of 369 diseases and injuries in 204 countries and territories, 1990-2019: a systematic analysis for the Global Burden of Disease Study 2019. The Lancet, 396(10258), pp.1204-1222.

Zellers, J.A., Christensen, M., Kjær, I.L., Rathleff, M.S. and Silbernagel, K.G., 2019. Defining components of early functional rehabilitation for acute Achilles tendon rupture: a systematic review. Orthopaedic journal of sports medicine, 7(11), p.2325967119884071.

Zhao, J.G., Meng, X.H., Liu, L., Zeng, X.T. and Kan, S.L., 2017. Early functional rehabilitation versus traditional immobilization for surgical Achilles tendon repair after acute rupture: a systematic review of overlapping metaanalyses. Scientific reports, 7(1), pp.1-7. 